\title{
Transurethral Resection of the Prostate (TURP): About 146 Cases at Sheikh Zayed Hospital in Nouakchott Mauritania
}

\author{
Mouhamadou Diagana, Yahya Tfeil, Mohamed Mahmoud Boya, Mohamed Bechir Essalem \\ Faculty of Medicine, Al Asyria University, Sheikh Zayed Hospital, Nouakchott, Mauritania \\ Email: bagdiagana@yahoo.fr, tefeil2000@gmail.com, mahmoudboya4@gmail.com, bechirely3@gmail.com
}

How to cite this paper: Diagana, M., Tfeil, Y., Boya, M.M. and Essalem, M.B. (2021) Transurethral Resection of the Prostate (TURP): About 146 Cases at Sheikh Zayed Hospital in Nouakchott Mauritania. Open Journal of Urology, 11, 518-524. https://doi.org/10.4236/oju.2021.1112052

Received: February 12, 2021

Accepted: December 27, 2021

Published: December 30, 2021

Copyright ( 2021 by author(s) and Scientific Research Publishing Inc. This work is licensed under the Creative Commons Attribution International License (CC BY 4.0).

http://creativecommons.org/licenses/by/4.0/

\begin{abstract}
Introduction: Transurethral resection of the prostate (TURP), entered our service in October 2015, since then it became a part of the service daily activity. The aim of our study is to assess the acceptance of the technic by our patients and by the nursing staff and to analyze the results of our initial experience. Materials and Methods: Retrospective study over 14-month (October 2015 to December 2016); bringing together all the patients who received TURP. The inclusion criteria are to have benefited from a TURP in our service. The exclusion criteria all patients operated by other techniques. It was a monopolar resection of the prostate using a glycocolle loop, the size limit of the prostate was $65 \mathrm{cc}$ or less. A questionnaire on the knowledge of the technique and its acceptance is made. The parameters studied the age of the patient; surgical indications; the course of the operation, the complications and the course. The frequency of TURP compared to other techniques and the demand for this technique by patients. The urinary catheter was left in place for 4 days, the patients were seen again on day 7 after the catheter was removed to assess the urine flow, a question regarding the opinion of the nursing staff was included and analyzed separately. Results: The total number of patients was 146; the mean age was 68.2 years. The main cause of consultation was urine retention $65 \%$. The average duration of the intervention was 56 minutes. The average length of hospital stay was 28 hours. The urinary catheter was removed on day 4 . Complications were bleeding in $7 \%$ of cases requiring blood transfusions. Failure to remove the indwelling catheter leads to revision via the transurethral route. The TURP syndrome was observed in 2 patients in our early patients but quickly resolved. The outcome was favorable in $75 \%$ of cases. Post operating management give satisfaction for nurse and acceptance was a success. Conclusion: TURP is now a routine surgical activity; it seems to have an upright place for BPH in our department; its impact on morbidity was remarkable.
\end{abstract}




\section{Keywords}

Prostate, Resection, Endoscopy, Mauritania

\section{Introduction}

Transurethral resection of the prostate (TURP) is the gold standard for the surgical treatment of benign prostatic hyperplasia. This technique entered our service in August 2015. It requires an investment in endoscopic equipment and a trained surgical team. We are postponing our early experiment in a hospital on the outskirts of Nouakchott. The objective of our study was to assess the acceptability of the technique, our results at the start of the experiment and the prospects for improvement. To introduce this treatment was a challenge for hospital where it never uses urological endoscopic before, for medical staff to have new habitude for managing patient and material, for patients who wait results of the new technique. Perspective is to do more minimally invasive for urological treatment in our department.

The introduction of this technique was a challenge for our hospital which did not perform minimally invasive surgery. The challenge for the healthcare team is to perfect themselves in new operating techniques.

The prospects are the generalization of minimally invasive surgery while urologic surgery.

\section{Materials and Methods}

It is a retrospective analytical study of the first 14-month from August 2015 to December 2016 (initial experience). The study setting was the urology department at Cheikh Zayed Hospital in Nouakchott. The department has 14 beds and four urologist surgeons at this time of the study. This establishment is located in the southern suburbs of the capital Nouakchott in a densely populated area with modest socio-economic levels. This was a monopolar loop resection using glycocolle. The indwelling catheter was kept in place for four days. The result was assessed one week and three weeks after the catheter was removed. Favorable outcome for patients with a good voiding stream without residue, failure if signs of dysuria persist. All patients operated on by TURP during this period are included, the exclusion criteria were resections of bladder masses and adénomectomies by conventional surgery. The parameters were age, operating time, bleeding, complications, the burden of postoperative care in comparison with adenomectomy techniques and the evaluation of the adherence of nursing staff to the technique by a questionnaire. All nurses to our department between operating room and hospitalization are selected: total 24 nurses (nurses to surgery room, 16 nurses from hospitalization rooms). Questions were: Difference between TURP and open surgery of prostate, how is your charge of work between them, how is patient's pain between then. What are your preference between 
them? Degrees of satisfaction about this new technique in our department to prove the capacity of adaptation to the minimally invasive surgery for medical team and for patients.

All nurses in the service were included in the study. A total of 24 nurses including 8 in the operating room and 16 in hospitalization. Questions about adaptability to the new procedure, the difference in post-operative care and staff satisfaction.

The statistic program is IPSS.

\section{Results}

A total of 146 patients were collected, the average age was 68.2 years with extremes ranging from 53 years to 89 years (Figure 1). The reason for consultation was dominated by urinary retention (UR) in $62 \%$ cases and signs of the lower urinary tract (SUBA) in 35\% (Figure 2).

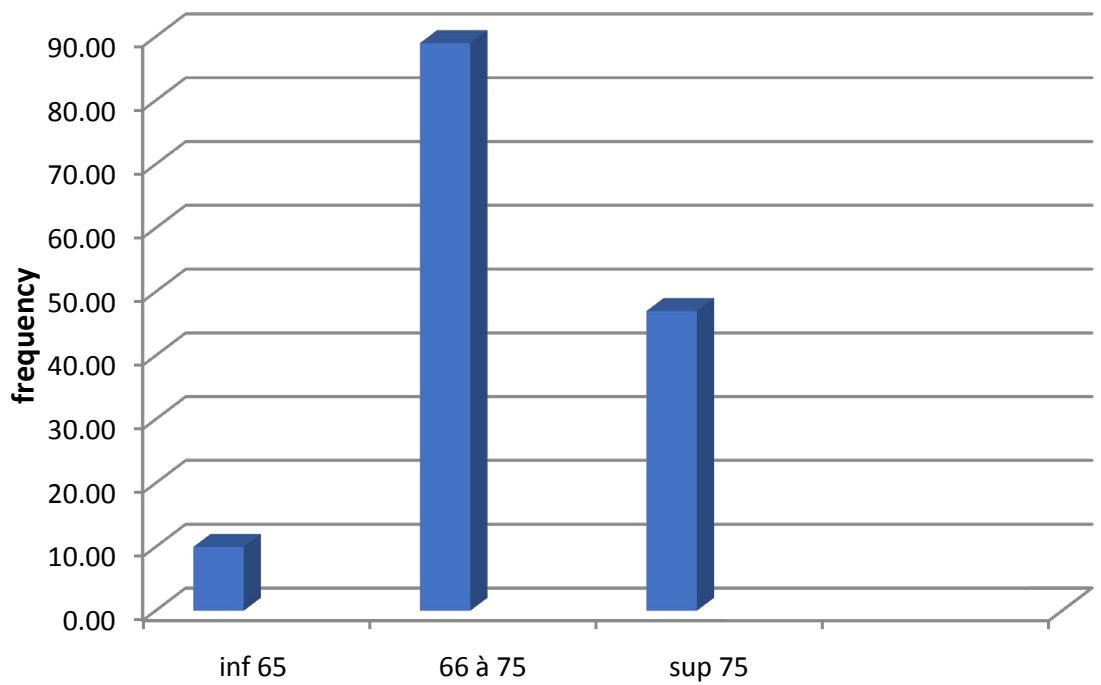

Figure 1. Distribution according to age.

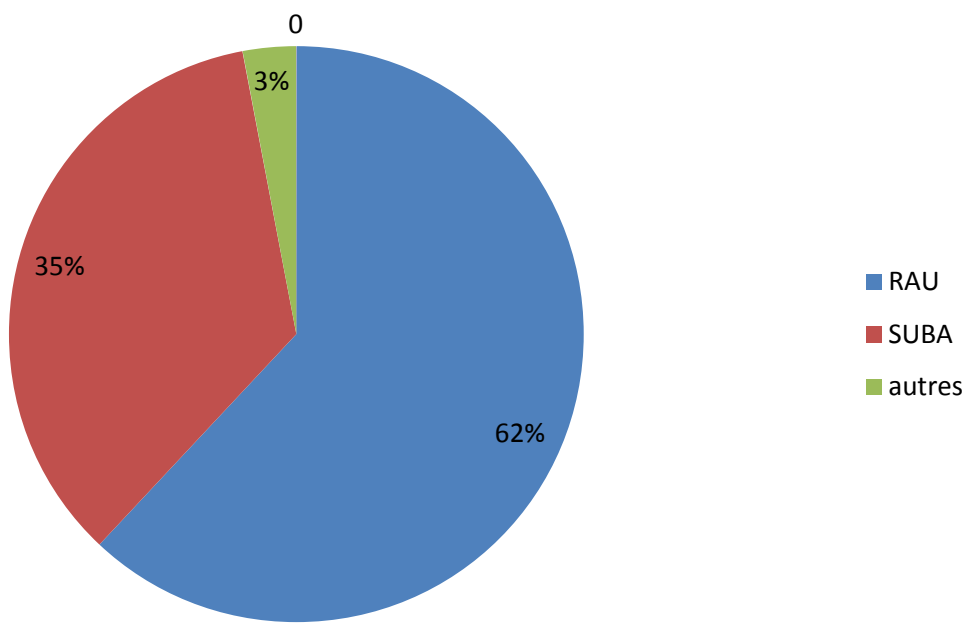

Figure 2. Distribution according to the reason for consultation. 
The average duration of the intervention was 56 minutes with extremes ranging from 41 minutes to 1 hour 10 minutes; the irrigation liquid for monopolar resection was glycocoll at a rate of 6 liters on average per patient. The average length of hospitalization was 28 hours with a maximum duration of 3 days. The urinary catheter was removed on Day 4 in the majority of cases. One procedure was stopped by abdominal distension following a bladder perforation. A blood transfusion in 7 of our patients who had a hemoglobin level of less than $9 \mathrm{~g} / \mathrm{dl}$ postoperatively. TURP syndrome was found in 2 cases with good progress after correction of the fluid and electrolyte disturbances. Urine retention in a patient who required a second look because resection shavings remained in the bladder. The adherence of the nursing staff was satisfactory in $72 \%$ of the cases, the reduction in the workload was $25 \%$ to $30 \%$ less compared to an adenomectomy by conventional surgery. The biggest difficulty for the nursing team was the maintenance of endoscopic equipment.

A significant immunization of the bladder washes liquor of $40 \%$ on average. The occurrence of bladder clotting decreases by more than $50 \%$. The use of post-operative antagonists by $42 \%$ and the reduction in the number of patients for dressings significantly (Table 1 ).

\section{Discussion}

In our study of the first 14 months of the start of our experiment; TURP represented 146 patients (29\% of all prostate surgery). This result reflects our patients' rapid adherence to the technique. Kane et al. [1] in Senegal had 84 cases in 2 years and Diakité [2] in Mali had 344 over 4 years. The average prostate volume was 60 grams. The length of hospital stay was drastically reduced with an average of 28 hours, in fact the patients were released on Dayl postoperatively with their indwelling catheter without any complications. According to the authors, this duration varies from 1 to 5 days [3] [4] [5]. The length of hospital stay increased for those who experienced bleeding and required blood transfusions. Kane et al. [1] had found 3.2 days. The urinary catheter is removed on Day 4 postoperative, the nursing workload in postoperative care had decreased considerably, justified by the reduction in postoperative clotting. Mamoulakiss et al. [6] estimated this reduction in the burden of post-operative care by $25 \%$ to $30 \%$. Bladder clotting and postoperative pain are less than open adenomectomy [4] [6]. The postoperative bleeding remains high at $7 \%$ requiring blood transfusions. Kane et al. [1] found 10\% bleeding compared to 5\% for Rassweiller et al. [7]. The

Table 1. Questions to nurses to compare care in prostatic adenomectomy and TURP.

\begin{tabular}{cccccc}
\hline Setting & $\begin{array}{c}\text { Bladder wash } \\
\text { Fluid }\end{array}$ & $\begin{array}{c}\text { Bladder } \\
\text { clotting }\end{array}$ & Pain & $\begin{array}{c}\text { Patient } \\
\text { dressing }\end{array}$ & $\begin{array}{c}\text { Preferred } \\
\text { procedure }\end{array}$ \\
\hline $\begin{array}{c}\text { Prostate } \\
\text { adenomrctomy }\end{array}$ & 24 liters & $95 \%$ & $100 \%$ & $100 \%$ & $25 \%$ \\
TURP & 9 liters & $50 \%$ & $42 \%$ & $48 \%$ & $75 \%$ \\
\hline
\end{tabular}


bleeding had significantly decreased in the series using exclusive bipolar resection [8] [9]. TURP syndrome was found in 2 patients with effective correction, these results are similar to those of Diakité in Mali [2]. TURP syndrome is the most complication with monopolar resection [10]; Bladder perforation and the long duration of the procedure, are the main causes of TURP syndrome [11] [12]. Retention by residual chips in the bladder has been reported from $0.5 \%$ to $1 \%$ of cases [13] [14]. Two patients presented with bleeding from pressure ulcers on day 7 and day 9 postoperatively. Bleeding from falling eschar was more frequent in large prostate and extensive use of the ball coagulation loop [7] [15]. Bleeding and turp syndrome are less in bipolar resection [16]. Postoperative pain was found in $40 \%$ of our patients, this pain was linked to the large caliber of the sheath for tight urethra [17]. The infectious complications described by Alsaywid et al. [18], should encourage obtaining a sterile ECBU before the surgical procedure. Postoperative progress and complications are rare in our series due to the relatively short follow-up. The authors describe post-resection stenosis and sclerosis of the prostatic compartment responsible for urinary disorders [19] [20]. Postoperative quality of life: urinary incontinence, erectile dysfunction and retroded ejaculation are significantly reduced in TURP [7]. TURP was recommended by $75 \%$ of nurses in our study. This recommendation reaches $100 \%$ in the literature [21]. The rapid return of patients to their activity is on average 2 weeks against 4 weeks for open-air surgery. The resumption of activity varies between 10 and 21 days depending on the authors [22].

\section{Conclusion}

The introduction of the TURP in our service had considerably improved our technical platform. The Strong adherence from nursing staff and patients, results in terms of reduction of post-operative care are significant. The postoperative quality of life and the rapid resumption of activity and maintenance of the factors encourage patients. This study will be completed by the results of the bipolar resection recently introduced in our service.

\section{Conflicts of Interest}

The authors declare no conflicts of interest regarding the publication of this paper.

\section{References}

[1] Kane, R., Ndoye, A., Niang, L., Barry, M., Labou, I., Jalloh, M., Ndoye, M. and Gueye, S.G. (2011) Résection transurétrale pour hypertrophie bénigne de la prostate au Sénégal. $A J U, 5,3$.

[2] Diakite, M.L., Berthe, H.J.G. and Diallo (2016) La résection endoscopique bipolaire: Expérience du service d'urologie du point G au Mali. Revue Africaine d Urologie et d'Andrologie, 1, 6.

[3] Delongchamps, N.B., Robert, G., Descazeaud, A., Cornu, J.N., Azzouz, A.R., Haillot, O., et al. (2012) Traitement de l'hyperplasie bénigne de prostate par techniques en- 
doscopiques électriques et adénomectomie voie haute: Revue de littérature du CTMH de l'AFU. Progrès en Urologie, 22, 73-79.

https://doi.org/10.1016/j.purol.2011.07.005

[4] Horchani, A., Binous, M.Y., Ben Hamida, A., Sallami, S., El Adbi, H. and Naji, A. (2007) Prevalence of Benign Prostatic Hyperplasia in General Practice and Practical Approach of the Tunisian General Practitioner. La Tunisie Medicale, 85, 619-624.

[5] Issa, M.M. (2008) Technological Advances in Transurethral Resection of the Prostate: Bipolar versus Monopolar TURP. Journal of Endourology, 22, 1587-1589. https://doi.org/10.1089/end.2008.0192

[6] Mamoulakis, C., Ubbink, D.T. and de la Rosette, J.J. (2009) Bipolar versus Monopolar Transurethral Resection of the Prostate: A Systematic Review and Meta-Analysis of Randomized Controlled Trials. European Urology, 56, 798-809. https://doi.org/10.1016/j.eururo.2009.06.037

[7] Rassweiler, J., Teber, D., Kuntz, R. and Hofmann, R. (2006) Complications of Trans Urethral Resection of the Prostate (TURP)-Incidence, Management, and Prevention. European Urology, 50, 969-980. https://doi.org/10.1016/j.eururo.2005.12.042

[8] Qu, L., Wang, X., Huang, X., Zhang, Y. and Zeng, X. (2008) The Hemostatic Properties of Transurethral Plasmakinetic Resection of the Prostate: Comparison with Conventional Resectoscope in an ex Vivo Study. Urologia Internationalis, 80, 292-295. https://doi.org/10.1159/000127344

[9] Ho, H.S. and Cheng, C.W. (2008) Bipolar Transurethral Resection of Prostate: A New Reference Standard? Current Opinion in Urology, 18, 50-55. https://doi.org/10.1097/MOU.0b013e3282f194db

[10] Fagerström, T., Nyman, C.R. and Hahn, R.G. (2010) Bipolar Transurethral Resection of the Prostate Causes Less Bleeding than the Monopolar Technique: A Single-Centre Randomized Trial of 202 Patients. BJU International, 105, 1560-1564. https://doi.org/10.1111/j.1464-410X.2009.09052.x

[11] Huang, X., Wang, L., Wang, X.H., Shi, H.B., Zhang, X.J. and Yu, Z.Y. (2012) Bipolar Transurethral Resection of the Prostate Causes Deeper Coagulation Depth and Less Bleeding than Monopolar Transurethral Prostatectomy. The Journal of Urology, 80, 1116-1120. https://doi.org/10.1016/j.urology.2012.07.024

[12] Tefekli, A., Muslumanoglu, A.Y., Baykal, M., Binbay, M., Tas, A. and Altunrende, F. (2005) A Hybrid Technique Using Bipolar Energy in Transurethral Prostate Surgery: A Prospective, Randomized Comparison. The Journal of Urology, 174, 1339-1343. https://doi.org/10.1097/01.ju.0000173075.62504.73

[13] Ho, H.S., Yip, S.K., Lim, K.B., Fook, S., Foo, K.T. and Cheng, C.W. (2007) A Prospective Randomized Study Comparing Monopolar and Bipolar Transurethral Resection of Prostate Using Transurethral Resection in Saline (TURIS) System. European Urology, 52, 517-522. https://doi.org/10.1016/j.eururo.2007.03.038

[14] Seckiner, I., Yesilli, C., Akduman, B., Altan, K. and Mungan, N.A. (2006) A Prospective Randomized Study for Comparing Bipolar Plasma Kinetic Resection of the Prostate with Standard TURP. Urologia Internationalis, 76, 139-143. https://doi.org/10.1159/000090877

[15] Ghozzi, S., Ghorbel, J., Ben Ali, M. and Dridi, M. (2014) Bipolar and the Coagulation Depth Achieved with Mono- and versus Monopolar Transurethral Resection of the Bipolar Transurethral Resection of the Prostate. Progrès en Urologie, 24, 121-126.

[16] Erturhan, S., Erbagci, A., Seckiner, I. and Yagci, F. (2007) Plasmakinetic Resection of the Prostate versus Standard Transurethral Resection of the Prostate: A Prospec- 
tive Randomized Trial with 1-Year Follow-Up. Prostate Cancer and Prostatic Diseases, 10, 97-100. https://doi.org/10.1038/sj.pcan.4500907

[17] Michielsen, D.P., Debacker, T., De Boe, V., Van Lersberghe, C., Kaufman, L., Braeckman, J.G., et al. (2007) Bipolar Transurethral Resection in Saline-An Alternative Surgical Treatment for Bladder Outlet Obstruction? The Journal of Urology, 178, 2035-2039. https://doi.org/10.1016/j.juro.2007.07.038

[18] Alsaywid, B.S. and Smith, G.H. (2013) Antibiotic Prophylaxis for Transurethral Urological Surgeries: Systematic Review. Urology Annals, 5, 61-74. https://doi.org/10.4103/0974-7796.109993

[19] Mayer, E.K., Kroeze, S.G., Chopra, S., Bottle, A. and Patel, A. (2012) Examining the "Gold Standard": A Comparative Critical Analysis of Three Consecutive Decades of Monopolar Transurethral Resection of the Prostate (TURP) Outcomes. BJU International, 110, 1595-1601. https://doi.org/10.1111/j.1464-410X.2012.11119.x

[20] Reich, O., Gratzke, C., Bachmann, A., Seitz, M., Schlenker, B. and Hermanek, P. (2008) Morbidity, Mortality and Early Outcome of Transurethral Resection of the Prostate: A Prospective Multicentre Evaluation of 10,654 Patients. The Journal of Urology, 180, 246-249. https://doi.org/10.1016/j.juro.2008.03.058

[21] Rassweiler, J., Teber, D., Kuntz, R. and Hofmann, R. (2006) Complications of Transurethral Resection of the Prostate (TURP) Incidence, Management, and Prevention. European Urology, 50, 969-979.

https://doi.org/10.1016/j.eururo.2005.12.042

[22] Hwang, E.C., Jung, S.I., Kwon, D.D., Lee, G., Bae, J.H., Na, Y.G., et al. (2014) A Prospective Korean Multicenter Study for Infectious Complications in Patients Undergoing Prostate Surgery: Risk Factors and Efficacy of Antibiotic Prophylaxis. Journal of Korean Medical Science, 29, 127. https://doi.org/10.3346/jkms.2014.29.9.1271

\section{Abbreviation}

TURP: Transurethral resection of the prostate $\mathrm{BPH}$ : Benign hypertrophy of prostate 